\title{
Solar Activity and Cloud Opacity Variations: A Modulated Cosmic-Ray Ionization Model
}

\author{
David Marsden \\ Scripps Institution of Oceanography \\ University of California, San Diego \\ 9500 Gilman Dr., Dept. 0242 \\ La Jolla, California 92093-0242 \\ email: dmarsden@ucsd.edu \\ Richard E. Lingenfelter \\ Center for Astrophysics and Space Sciences \\ University of California, San Diego
}

Received —

8/23/02 


\begin{abstract}
The observed correlation between global low cloud amount and the flux of high energy cosmic rays supports the idea that ionization plays a crucial role in tropospheric cloud formation. We explore this idea quantitatively with a simple model linking the concentration of cloud condensation nuclei to the varying ionization rate due to cosmic rays. Among the predictions of the model is a variation in global cloud optical thickness, or opacity, with cosmic-ray rate. Using the International Satellite Cloud Climatology Project database (1983-1999), we search for variations in the yearly mean visible cloud opacity and visible cloud amount due to cosmic rays. After separating out temporal variations in the data due to the Mt. Pinatubo eruption and El Niño/Southern Oscillation, we identify systematic variations in opacity and cloud amount due to cosmic rays. We find that the fractional amplitude of the opacity variations due to cosmic rays increases with cloud altitude, becoming approximately zero or negative (inverse correlation) for low clouds. Conversely, the fractional changes in visible cloud amount due to cosmic rays are only positively-correlated for low clouds and become negative or zero for the higher clouds. The opacity trends suggest behavior contrary to the current predictions of ion-mediated nucleation (IMN) models, but more accurate temporal modeling of the ISCCP data is needed before definitive conclusions can be drawn.
\end{abstract}




\section{Introduction}

The primary source of energy for the Earth's atmosphere is the Sun, so it is reasonable to explore whether changes in the global climate result from solar variability. It was first suggested by the astronomer William Herschel (Herschel 1801) that variations in the solar irradiance caused by sunspots could lead to climatic changes on Earth, and he cited the variation of British wheat prices with sunspot number as evidence for this link. The occurrence of the "Little Ice Age" during the 1645-1715 Maunder sunspot minimum (Eddy 1976), the correlation between the long-term solar cycle variations and tropical sea surface temperatures (Reid 1987), polar stratospheric temperatures (Labitske 1987), and the width of tree rings (Zhou and Butler 1998), along with many other studies also support a link between solar variations and the Earth's climate.

A direct link between the Sun and these phenomena is tenuous, however, because the magnitude of the solar irradiance variation over the 11-year solar cycle is very small. Over the 1979-1990 solar cycle, for example, the variation in the irradiance was only $\sim 0.1 \%$ (Fröhlich 2000), or $\sim 0.3 \mathrm{~W} \mathrm{~m}^{-2}$ globally-averaged at the top of the atmosphere. This is insufficient to power the sea surface temperature changes associated with the solar cycle by a factor of $3-5$ (Lean 1997), and is significantly smaller than the globally-averaged forcings due to clouds ( $\sim 28 \mathrm{~W} \mathrm{~m}^{-2}$; e.g. Hartmann 1993), anthropogenic greenhouse gases $(\sim 2 \mathrm{~W}$ $\mathrm{m}^{-2}$; Wigley and Raper 1992), and anthropogenic aerosols $\left(\sim 0.3-2.0 \mathrm{~W} \mathrm{~m}^{-2}\right.$; Charlson et al. 1992; Kiehl and Briegleb 1993), suggesting that any direct atmospheric forcing from solar irradiance variations would be relatively unimportant.

An indirect link between solar cycle variations and the Earth's climate appears more likely, especially given the discovery of a link between the flux of Galactic cosmic rays (GCRs) and global cloudiness (Svensmark and Friis-Christensen 1997) in the ISCCP cloud database (Rossow and Schiffer 1999). The Sun modulates the GCR flux at the Earth 
through the action of the solar wind, which scatters and attenuates the GCRs in times of heightened solar activity (solar maximum; e.g. Jokipii 1971). Using $3.7 \mu \mathrm{m}$ infrared (IR) cloud amounts from the ISCCP database for the years 1983-1993, Marsh and Svensmark (2000) and Pallé Bagó and Butler (2000) showed that there is evidence of a positive GCR-cloud correlation only for low $(<3 \mathrm{~km})$ clouds, and that the effect of the cosmic rays on global cloud amount appears to be greatest at the low to mid latitudes. The globally-averaged forcing due to the increase in low clouds associated with the solar cycle GCR variations is estimated (Kirkby and Laaksonen 2000) to be approximately $-1.2 \mathrm{~W}$ $\mathrm{m}^{-2}$, which is sufficient to power the sea surface temperature variations (Lean 1997). This is also comparable in magnitude (but opposite in sign) to the forcing due to anthropogenic $\mathrm{CO}_{2}$ emission over the last century (Svensmark and Friis-Christensen 1997). Decreasing local cloud amounts correlated with short-term Forbush decreases in cosmic-ray rates were observed by Pudovkin and Veretenko (1995).

The reality of the GCR-cloud connection has been questioned by a number of authors (Kernthaler, Toumi, and Haigh 1999; Jorgensen and Hansen 2000; Norris 2000). These objection can be distilled into three main points: 1) The GCR-cloud correlation should be seen prominently in high (cirrus) clouds at high latitudes where the cosmic-ray intensity is highest, 2) the increased cloudiness can be more plausibly attributed to other phenomena instead of GCRs, and 3) the correlation is an artifact of the ISCCP analysis. The first objection is addressed by the theory of ion-mediated nucleation (IMN: Yu and Turco 2001; $\mathrm{Yu} 2002$ ), in which the efficiency of the cosmic-ray interaction is limited at high altitudes by the lack of aerosol precursor vapors such as $\mathrm{H}_{2} \mathrm{SO}_{4}$ relative to the ion concentration. For the second objection, the temporal profile of the GCR-cloud correlation may be inconsistent with the profiles of the dominant volcanic and El Niño/Southern Oscillation (ENSO) events during the same time period ( Kirkby and Laaksonen 2000), although no quantitative study of the various temporal signatures in the data has been undertaken. Finally, the ISCCP 
artifacts pointed out by Norris (2000) are troubling, but it is not clear that they are of sufficient magnitude to produce the observed GCR-cloud correlation, and it doesn't explain why the correlation exists only for low clouds and not the other cloud types in the ISCCP database.

The linkage between cosmic rays and cloud formation has been recently investigated by a number of authors (Yu 2002; Yu and Turco 2001; Tinsley 2000 and references therein). Here we apply a perturbative approach to quantify the effects of variations in the cosmic-ray rate on the optical thicknesses, or opacities, of clouds, and use the observed cloud opacity variations to constrain the microphysical models of ion-mediated ultrafine particle formation. The paper is organized as follows. In the next section we discuss how the effect of cosmic rays could alter the optical thickness and emissivity of clouds by affecting the nucleation of condensation nuclei $(\mathrm{CN})$. The search for variations in cloud optical properties using the ISCCP database and their correlation with cosmic-ray flux variations are discussed in Section 3. A discussion of the results is given in Section 4., and finally we summarize our results in Section 5 .

\section{Effects of GCRs on Cloud Properties}

\subsection{Nucleation}

Cosmic rays form water droplets in the supersaturated air of a classical cloud chamber (Wilson 1901), and it seems plausible that they could also play a significant role in natural cloud formation. Yu and Turco $(2000,2001)$ and Yu (2002) have investigated the formation

of ultrafine $\mathrm{CN}$ from charged molecular clusters formed from cosmic-ray ionization, and they find that the charged clusters grow more rapidly and are more stable than their neutral counterparts up to a size of $\sim 10 \mathrm{~nm}$. Although the subsequent growth of the 
cosmic-ray formed ultrafine $\mathrm{CN}$ to viable $\sim 100 \mathrm{~nm}$ cloud condensation nuclei $(\mathrm{CCN})$ has not been explored, the concentration of $\mathrm{CCN}$ should also reflect the $\mathrm{CN}$ concentration, as well as the direct influence of cosmic rays, if the cosmic-ray ionization rate does not affect other important nucleation efficiency parameters such as condensible vapor concentration, temperature, and pressure. We will make this assumption here although it may not be strictly true with respect to the condensible vapor concentration (see e.g. Turco, Yu, and Zhao 2000; Yu 2002).

Althought the formation of CCN and ultimately cloud droplets is a function of many variable factors such as temperature, pressure, vapor concentration, and relative humidity, we can quantify the effects of small variations in the ionization rate (primarily due to cosmic rays above ocean and at altitudes $>1 \mathrm{~km}$ above land; e.g. Reiter 1992) on the number of CCN through a perturbation approach, i.e.

$$
N_{\mathrm{CCN}}(q+\Delta q, V) \approx N_{\mathrm{CCN}}(q, V)+\left.\Delta q \frac{\partial N_{\mathrm{CCN}}}{\partial q}\right|_{V}
$$

where $N_{\mathrm{CCN}}$ is the concentration of $\mathrm{CCN}, q$ is the ionization rate, $V$ refers to the set of parameters other than the ionization rate affecting $N_{\mathrm{CCN}}$, and the partial derivative is evaluated for fixed $V$ (hereafter this will not be written explicitly). Along with the assumption discussed previously, this approach assumes that the quantity $\Delta q\left|\partial N_{\mathrm{CCN}} / \partial q\right|<<N_{\mathrm{CCN}}(q, V)$, which is probably true for solar cycle variations, where $q$ typically varies by $<30 \%$, but may not be true during periods of large scale changes in the geomagnetic field (e.g. Tric et al. 1992).

To quantify the effect of varying CCN concentrations on cloud optical thicknesses, we envision the two idealized scenarios depicted in Figure 1. In both cloud formation scenarios, changes in the ionizing cosmic-ray flux cause changes in the number of cloud condensation nuclei through the process of ion-mediated nucleation on the formation of ultrafine CN 
in accordance with the assumptions mentioned abovel. In the first case we assume that the nucleation of cloud droplets is limited by the available amount of water in the supersaturated air, so that the liquid water content (LWC), or density of water in droplets, is constant. Therefore the amount of water per droplet and hence the effective radii of cloud droplets will change with the cosmic-ray ionization rate. This is analogous to the "Twomey Effect" of enhanced aerosol pollution on droplet size distributions and the albedo of clouds (Twomey 1977; Rosenfeld 2000), and would primarily occur in environments where the amount of water in the air (and not the number of $\mathrm{CCN}$ ) is the limiting factor. Thus, using (1), we would expect that the effective radius $R_{\text {eff }}$ of the cloud droplet distribution resulting from a small change in the cosmic-ray ionization rate $\Delta q$ in any particular volume of air will be

$$
R_{e f f}=\left[\frac{N_{\mathrm{CCN}}(q, V)}{N_{\mathrm{CCN}}(q+\Delta q, V)}\right]^{1 / 3} R_{e f f}^{0} \approx\left(1+\frac{\Delta q}{N_{\mathrm{CCN}}} \frac{\partial N_{\mathrm{CCN}}}{\partial q}\right)^{-1 / 3} R_{e f f}^{0}
$$

where $R_{e f f}^{0}$ is the effective radius of the unperturbed droplet distribution, which we will associate with the solar maximum period of the solar cycle.

In the second case in Figure 1, we assume that the change in CCN concentration resulting from change in cosmic-ray ionization causes a proportionate change in the amount of water extracted from the supersaturated air, with the effective radius of the cloud droplet distribution remaining constant. This is the case where the formation of the cloud is limited by the local availability of $\mathrm{CCN}$ and not condensible water. This effect has been seen in the marine boundary layer in ship track clouds (Conover 1966), which have higher reflectivities (Coakley, Bernstein, and Durkee 1987) and liquid water contents (Radke, Coakley, and King 1989) due to the formation of additional ultrafine CN from ship exhaust. The perturbed

\footnotetext{
${ }^{1}$ We have assumed $\partial N_{\mathrm{CCN}} / \partial q>0$ in Figure 1 , which need not be valid for all $q$.
} 
liquid water content of a cloud in any particular volume of air will then be given by

$$
\mathrm{LWC} \approx\left(1+\frac{\Delta \mathrm{q}}{\mathrm{N}_{\mathrm{CCN}}} \frac{\partial \mathrm{N}_{\mathrm{CCN}}}{\partial \mathrm{q}}\right) \mathrm{LWC}_{0},
$$

where $\mathrm{LWC}_{0}$ is the unperturbed cloud liquid water content associated with solar maximum as before. These two scenarios probably represent extremes of the direct cosmic-ray ionization effect on the clouds. As in the ship track clouds, the effect of the GCRs will probably be a combination of both LWC changes and $R_{\text {eff }}$ changes, with the magnitude of the effect being bounded by the changes given in (2) and (3).

\subsection{Radiative Properties}

Changes in the cloud liquid water content and droplet effective radius, associated with changes in the ionization rate due to cosmic rays, will result in changes in cloud opacities. The optical thickness $\tau$ of a uniform cloud layer of thickness $\Delta z$ is given by (van den Hulst 1981):

$$
\tau=\Delta z \int_{0}^{\infty} Q_{e x t} n(r) \pi r^{2} d r,
$$

where $n(r) d r$ is the concentration of cloud droplets with radii between $r$ and $r+d r, Q_{e x t}$ is the Mie extinction efficiency, and it is commonly assumed that

$$
\frac{\int_{0}^{\infty} Q_{e x t} n(r) r^{2} d r}{\int_{0}^{\infty} n(r) r^{2} d r}=2,
$$

which is a good approximation when $2 \pi r / \lambda>>1$, where $\lambda$ is the wavelength (Stephens 1984).

The effective radius of the cloud droplet distribution is given by

$$
R_{e f f}=\frac{\int_{0}^{\infty} n(r) r^{3} d r}{\int_{0}^{\infty} n(r) r^{2} d r}
$$


and the cloud liquid water content is given by

$$
\mathrm{LWC}=\frac{4}{3} \pi \rho \int_{0}^{\infty} \mathrm{n}(\mathrm{r}) \mathrm{r}^{3} \mathrm{dr}
$$

where $\rho$ is the density of liquid water. Combining these equations, we see that

$$
\tau \approx \frac{3}{2} \frac{\mathrm{LWC} \Delta z}{\rho R_{e f f}} .
$$

Thus from (8) we would expect that an increase (decrease) in the mean $R_{\text {eff }}$ and a decrease (increase) in the mean LWC, resulting from ionization variations due to cosmic rays, would result in a decrease (increase) the mean opacity of clouds.

The change in cloud opacity with cosmic-ray rate can be quantified using the perturbation assumptions discussed in Section 2.1. and equations (2), (3), and (8). The fractional change in cloud opacity is then given by

$$
\frac{\delta \tau}{\tau} \sim \frac{\Delta q}{f N_{\mathrm{CCN}}} \frac{\partial N_{\mathrm{CCN}}}{\partial q},
$$

where $f=1(3)$ for $\mathrm{CCN}$ (water) limited cloud formation, and the fractional change in the perturbed opacity $\tau$ (relative to the unperturbed opacity $\tau_{0}$ ) is defined by $\delta \tau / \tau=\left(\tau-\tau_{0}\right) / \tau_{0}$. As mentioned previously, this derivation assumes that the right hand side of (9) is much less than one, which may not be the case for large changes in $q$ and $N_{\mathrm{CCN}}$. As before we will assume that the unperturbed (perturbed) values of $q$ and $N_{\mathrm{CCN}}$ refer to the values at solar maximum (minimum).

At visible wavelengths from space, the primary consequence of the change in cloud opacity associated with cosmic rays will be an increase in cloud reflectivity, or albedo. To investigate this, we use the radiative transfer code SBDART (Ricchiazzi, Yang, and Gautier 1998) to calculate the top of the atmosphere broadband $(0.25-4.00 \mu \mathrm{m})$ upward flux for three uniform low cloud models: 1) a $1 \mathrm{~km}$ thick cloud layer extending to a height of $2 \mathrm{~km}$, 2) a $2 \mathrm{~km}$ thick cloud extending to a height of $3 \mathrm{~km}$, and 3 ) a $0.5 \mathrm{~km}$ cloud layer extending 
to $1.5 \mathrm{~km}$. These simulations were done with a tropical atmosphere profile McClatchey et al. 1972) and an ocean surface albedo. The fractional increases in albedo, resulting from a $10 \%$ increase in the number of cloud droplets due to cosmic ray ionization variations, is shown in Figure 2 for the $1 \mathrm{~km}$ thick cloud case, for a wide range of LWC and $R_{e f f}$ in the variable LWC case (top panel) and the variable $R_{\text {eff }}$ case (bottom panel). In both cases the contours of changing albedo approximately parallel the change in optical thickness calculated assuming $Q_{\text {ext }}=2.0$

Figure 3 shows the fractional change in albedo directly as a function of opacity for all three cloud models. This figure clearly shows that the change in albedo is largest for clouds with opacities $\tau$ between 1 and 10, but is roughly independent of cloud geometrical thickness. Figures 2 and 3 indicate that the change in cloud optical thickness can be used to quantify the effects of the cosmic rays on cloud optical properties. Although the fractional change in albedo due to the cosmic rays is only $\sim 2-5 \%$ for a $10 \%$ variation in the number of cloud droplets, this can produce a significant forcing per cloud of $\sim 7-16 \mathrm{~W} \mathrm{~m}^{-2}$ at the top of the atmosphere for a solar zenith angle of $40^{\circ}$. The modulation of cloud opacity due to cosmic rays could therefore produce a similar modulation of the Earth's energy budget over the 11 year solar cycle, although the exact amount of forcing due to cosmic rays will depend sensitively on cloud amount variations, cloud opacity variations, and the efficiency at which changes in the cosmic ray rate are reflected in the number of cloud condensation nuclei.

Because of the relationship between cloud opacity and emissivity, the cosmic rays should also produce an observable effect on cloud emission at infrared (IR) wavelengths. The effective IR emissivity $\epsilon$ can be parameterized by a relation of the form (Stephens 1978):

$$
\epsilon=1-\exp \left(-a_{0} \operatorname{LWC} \Delta z\right)
$$


where $a_{0}$ is the mass absorption coefficient. Empirical fits to IR emission from water clouds yield $a_{0}=0.130$ (Stephens 1978). The exponent in (10) is proportional to the cloud optical thickness for a given droplet effective radius, so the infrared emissivity increases with cloud opacity, with the change being most noticeable for optically thin clouds. Therefore one would expect a change in IR emission, along with the primary effect of changes in visible albedo, from clouds at solar minimum relative to clouds at solar maximum if the cosmic rays change the cloud liquid water contents. Interestingly, a correlation between cosmic ray rate and cloud top temperature for low clouds has been reported by Marsh and Svensmark (2000), supporting this hypothesis.

\section{Cloud Opacity Variations}

\subsection{ISCCP Data}

To search for systematic temporal changes in synoptic scale cloud optical properties, we used the International Cloud Climatology Project (ISCCP) monthly gridded cloud products ("D2") datasets, a compilation of cloud properties derived from satellite observations during the period 1983-1999 (Rossow and Schiffer 1999). The ISCCP D2 data used here consists of mean daytime cloud amount fractions and visible optical depths, as a function of time, for 6596 "boxes" with equal area covering the entire surface of the Earth. For a given time, the cloud amount fraction in each box is defined as the number of cloudy satellite image pixels, as determined by a cloud detection algorithm, divided by the total number of pixels in the box. The cloud optical thicknesses are derived from the visible satellite cloud albedos by using a radiative transfer model and assuming spherical droplets with droplet sizes characterized by a gamma distribution with variance 0.15 and $R_{e f f}=10 \mu \mathrm{m}$. ISCCP cloud top temperatures are simultaneously determined from the $3.7 \mu \mathrm{m}$ IR radiances, allowing 
for determination of cloud altitude and pressure, and the low, mid-level, and high clouds are defined as having cloud top pressures $P>680 \mathrm{mb}, 440<P<680 \mathrm{mb}$, and $P<440$ mb, respectively. Because we require the simultaneous visible and infrared radiances to determine the opacity and cloud height for our analysis, we only use the ISCCP daytime data. This is a different dataset than the diurnal 1983-1993 IR data used for the cloud amount analyses of Marsh and Svensmark (2000) and Pallé Bagó and Butler (2000).

Detailed information on the distribution of cloud optical thicknesses is not preserved in the the ISCCP D2 database, and instead the mean optical thickness $\bar{\tau}_{i}$ is recorded for three broad opacity bands $i$ : $0.0-3.6,3.6-23.0$, and $23.0-379.0$. Thus a detailed analysis of the change in $\tau$ over the solar cycle is not possible using the D2 data, but a value of the weighted mean cloud optical thickness $\bar{\tau}$ can be calculated using

$$
\bar{\tau}=\frac{\sum_{i=1}^{3} \bar{A}_{i} \bar{\tau}_{i}}{\sum_{i=1}^{n} \bar{A}_{i}},
$$

where the $\bar{A}_{i}$ are the total mean cloud amount fractions within each of the broad ISCCP optical thickness bins mentioned above. We calculated $\bar{\tau}$ separately for the three cloud altitude levels and for two latitude bands with $|\phi| \leq 40.0^{\circ}$ (low latitude) and $|\phi|>40^{\circ}$ (high latitude). The error associated with each $\bar{\tau}_{i}$ was estimated by calculating the standard deviation of each ISCCP data point, from the scatter about the mean, and scaling by the square root of the number of data points.

The mean optical thicknesses $\bar{\tau}$ as a function of time for the low latitude clouds are shown in Figure 4, and the corresponding result for global high latitude clouds is shown in Figure 5. Shaded is the two year period in which the effects of the Mt. Pinatubo eruption appear to be most significant. Also shown for comparison are the mean counting rates from the Climax, Colorado neutron monitor run by the University of Chicago (obtained from http://ulysses.uchicago.edu/NeutronMonitor/neutron_mon.html), which is a good measure of the local cosmic-ray ionization rate. In the low latitude case, the abrupt and large 
decrease in $\bar{\tau}$ during $1991-1993$ is due to the eruption of Mt. Pinatubo, and the subsequent plot scaling obscures smaller scale opacity variations. For comparison, we also plot the total

mean cloud amount fractions $A=\sum_{i=1}^{3} \bar{A}_{i}$ for the same two latitude bands in Figures 6 and 7. These plots show evidence for increases in mean cloud amount due to Mt. Pinatubo, as well as the smaller-scale temporal variations.

\subsection{Extracting The Cloud Variations Due to Cosmic-Rays}

To search for subtle variations in the ISCCP cloud opacities and amounts due to cosmic rays only, it is first necessary to eliminate the opacity variations in the data due to the Mt. Pinatubo volcanic eruption in June-September 1991 and strong ENSO events during the period of the ISCCP data. To separate out the various temporal signatures in the ISCCP data, we use a linear temporal model of the form

$$
F(t)=\sum_{k=0}^{3} b_{k} X_{k}(t),
$$

where $F(t)$ is the mean ISCCP quantity of interest for the year $t$, which for our purpose is either the visible cloud opacity $\bar{\tau}$ or the mean cloud amount/fractional area $A$. The model consists of four temporal basis vectors $X_{k}$, which are functions of time, each scaled by a linear coefficient $b_{k}$. For our temporal model we choose basis vectors corresponding to constant level of the given quantity $(k=0)$ and variations due to ENSO events (e.g. Kuang, Jiang, and Yung 1998), the Mt. Pinatubo eruption of 1991, and cosmic rays ( $k=1-3$, respectively). Given the functional form of the basis vectors, the best-fit values of the linear coefficients can be determined through least squares minimization, and the fractional change in the time-varying ISCCP quantity over the data stretch is then given by $\delta F / F=b_{k} / b_{0}$, where $k=1-3$. This model assumes a linear correlation between the quantity of interest and the basis vectors and assumes no time delays; more complicated models are possible but will not be considered here. 
The normalized basis vectors used in the temporal analysis of the ISCCP cloud data are shown in Figure 8. All of the vectors are scaled to values between zero and one. For the ENSO term $X_{1}$ we use the scaled yearly-averaged Southern Oscillation Index (SOI) from the Australian Bureau of Meteorology (obtained from http://www.bom.gov.au/climate/current/soihtm1.shtml). The SOI is a measure of the size of fluctuations in the sea level pressure difference between Tahiti and Darwin, Australia, and small values of the scaled SOI denote El Niño conditions and large values La Niña - both of which affect global weather (Rasmusson and Carpenter 1982). To parameterize the effect of the Mt. Pinatubo eruptions of 1991, we adopt a simple step function for $X_{2}$, with identical non-zero intensities only for years 1991 and 1992. For the final term in the temporal model, $X_{3}$, we use the scaled cosmic-ray rate from the Climax neutron monitor. Neutron monitor rates are directly proportional to the ionization rates due to cosmic rays because the neutrons are produced by the same cosmic ray cascade particles that produce the ionization, and the neutrons subsequently diffuse through less than $100 \mathrm{~m}$ of air before they are thermalized and captured by $\mathrm{N}$ to form ${ }^{14} \mathrm{C}$ (e.g. Lingenfelter, 1963). Neutron counters are thus unsusceptible to background ionizations due to terrestrial radiation from radioactive decays, which dominate the ionization signal from Galactic cosmic rays only below $\sim 1 \mathrm{~km}$ in the atmosphere (Reiter 1992).

The results of the temporal fitting of both the ISCCP visible cloud opacities and amounts are shown in Table 1. Formally most of the fits are not good, with reduced chi-squares ranging from $\sim 0.7-7.8$ for twelve degrees of freedom. There are a number of possible factors that could be contributing to this. For example the error bars on the data may have been underestimated, leading to an artificially large values of chi-squared. Another possibility is that our fitting model is missing other significant temporal drivers, or perhaps a non-linear model or different basis vectors may be required to fit the data. We tried to fit the ISCCP data with linear models composed of different combinations of our 
four basis vectors, and models with the cosmic-ray term provided a better fit to the data in general. Nevertheless it is possible that un-modeled phenomena mimic the temporal signature of cosmic rays in the data; more robust calculations of ISCCP error bars, inclusion

of more ISCCP data, and exploration of more complicated temporal models in future work will help resolve this issue.

The fractional variation in visible opacity $\delta \tau / \tau$ associated with the cosmic rays ranges from $\sim+10 \%$ for high clouds to $-7 \%$ for low clouds. For the mean visible cloud amounts the variation due to cosmic rays is just the opposite - becoming greater in magnitude as the cloud height decreases - qualitatively consistent with the positive correlation seen in the ISCCP IR data between cosmic ray rate and low clouds Svensmark and Friis-Christensen 1997; Marsh and Svensmark 2000; Pallé Bagó and Butler 2000). Therefore the high clouds appear to become thicker but smaller in response to increasing cosmic ray flux, while for the low clouds the response is the opposite.

\section{Discussion}

The observed variation of cloud optical thicknesses with cosmic-ray rate can be used to constrain microphysical models of the cloud condensation nuclei concentration $N_{\mathrm{CCN}}$ using (9). Of crucial importance is the partial derivative $\partial N_{\mathrm{CCN}} / \partial q$, which determines the sign of the change in opacity with cosmic-ray rate. Recently $\mathrm{Yu}(2002)$ calculated $N_{\mathrm{CCN}}$ as a function of altitude and ionization rate using an ion-mediated nucleation code. Given this model and the vertical profiles of sulfuric acid vapor concentration, ionization rate, temperature, relative humidity, pressure, and surface area of pre-existing particles assumed therein $(\mathrm{Yu} 2002)$, the value of $\partial N_{\mathrm{CCN}} / \partial q$ peaks at values of $q_{\text {peak }}=12,8$, and 4 ion pairs $\mathrm{cm}^{-3}$ for low, mid-level, and high clouds, respectively, such that $\partial N_{\mathrm{CCN}} / \partial q>0$ for $q<q_{\text {peak }}$ and $\partial N_{\mathrm{CCN}} / \partial q<0$ for $q>q_{\text {peak }}$. Using the cosmic-ray ionization rates found by 
Neher $(1961,1967)$ interpolated to geomagnetic latitude $40^{\circ}$, we find typical ionization rates of, respectively, $q \sim 3,8$, and 23 ion pairs $\mathrm{cm}^{-3}$ for the low, mid-level, and high ISCCP clouds. Therefore from (9) we would expect a positive or zero correlation between opacity and cosmic-ray rate only for low clouds, and negative correlations for higher clouds for this model. We observe just the opposite, but the precision of the temporal model fits to the ISCCP data is not sufficient for us to rule out the Yu (2002) model based on the data.

All three of the time-varying parameters in our temporal model show inverse correlations between mean visible cloud opacity and amount, suggesting a common origin for this behavior. These inverse correlations are illustrated in Figure 9. These are probably not artifacts of the averaging process because the quantities in Figure 9 have been normalized by their constant model terms in their temporal fits. One possible explanation for the inverse opacity-amount correlation is via a feedback mechanism. For the case of positive opacity variations, an increase in mean cloud opacity and albedo would result in increased energy loss to space and eventually less surface heating and subsequent water evaporation. Hence clouds would tend to be smaller and have smaller areas than they would otherwise. Conversely, for negative opacity variations clouds would tend to be larger. Global climate simulations (Chen and Ramaswamy 1996) indicate that global cloud albedo-increasing perturbations - similar to the changes induced by cosmic rays - decrease the global transport of moisture from the tropics, which then could conceivably produce fewer or smaller global clouds on average by this mechanism. Dynamical simulations of the response of global cloudiness to synoptic changes in the opacity are needed to investigate this.

\section{Summary}


Here we consider a model in which Galactic cosmic rays alter the optical properties of clouds by changing the number of available cloud condensation nuclei. The main observational consequence of our model is a change in mean cloud opacity, with a secondary effect being a change in infrared emittance for optically thin clouds due to the relationship between cloud emissivity and opacity. We use the global ISCCP cloud database to search for variations in cloud properties due to cosmic rays, and after subtracting the background signals in the data due to Mt. Pinatubo and ENSOs, we find systematic variations in both opacity and cloud amount associated with changes in the cosmic-ray rate. The fractional variation in opacity attains a maximum positive value for high clouds and decreases with height, becoming negative or zero for low clouds. The fractional variation of the cloud amounts with cosmic-ray rate, however, show the opposite trend - increasing from a negative correlation at high altitudes to a positive correlation at low altitudes, which is consistent with the positive correlation between global low clouds clouds and cosmic-ray rate seen in the infrared (Svensmark and Friis-Christensen 1997; Marsh and Svensmark 2000; Pallé Bagó and Butler 2000)

Clearly more work is needed to model the opacity and cloud amount variations seen in the ISCCP data. Using our simple temporal model and perturbative approach, we have outlined a framework on which the variations in the data due to cosmic rays can be isolated and compared to model predictions. As the time span of the ISCCP data increases in length, more complicated models with additional components and nonlinear dependencies can be used, and the analysis can then be more robust. The ISCCP data requires the culling together and normalizing of many disparate satellite datasets (Rossow and Schiffer 1999), and although this approach is necessary at the present time it is not ideal. One complement to the ISCCP global cloud data would be provided by the NASA deep space mission Triana, which would be able to retrieve cloud optical thicknesses simultaneously over the entire sunlit Earth from the L1 Lagrangian point between the Earth and the Sun. 
Continuous deep space observing of Earth's clouds would be ideal for detecting not only the solar cycle variations seen here but also the shorter duration but possibly more frequent variations in global cloud cover associated with Forbush decreases of Galactic cosmic rays and high energy solar proton events from the Sun.

We thank the AVANTI article service of the Scripps Institution of Oceanography Library, and acknowledge the use of cosmic-ray data from the University of Chicago (National Science Foundation grant ATM-9912341) and Southern Oscillation Index data from the Australian Bureau of Meteorology. We also would like to thank the anonymous referees for very helpful comments. 


\section{REFERENCES}

Charlson, R. J., et al. 1992: Climate forcing by anthropogenic aerosols. Science, 255, 423-429.

Chen, C.-T., and V. Ramaswamy 1996:, Sensitivity of simulated global climate to perturbations in low cloud microphysical properties. Part I: global perturbations. J. Climate, 9, 1385-1402.

Coakley, J. A. Jr., R. L. Bernstein, and P. A. Durkee 1987: Effect of Ship-Stack Effluents on Cloud Reflectivity. Science, 237, 1020-1022.

Conover, J. H. 1966: Anomalous cloud lines. J. Atmos. Sci., 23, 778-785.

Eddy, J. A. 1976: The Maunder minimum. Science, 192, 1189-1202.

Fröhlich, C. 2000: Observations of irradiance variations. Spa. Sci. Rev., 94, 15-24.

Hartmann, D. L. 1993: Radiative effects of clouds on Earth's climate. Aerosol-Cloud-Climate Interactions, P. V. Hobbs, Ed., Academic Press Inc., 151-155.

Herschel, W. 1801: Observations tending to investigate the nature of the Sun, in order to find the causes or symptoms of its variable emission of light and heat; with remarks on the use that may possible be drawn from solar observations. Phil. Trans. Royal Soc. Lon., 91, 265-318.

Hoppel, W. A. 1985: Ion-aerosol attachment coefficients, ion depletion, and the charge distribution on aerosols. J. Geophys. Res., 90, 5917-5923.

Jokipii, J. R. 1971: Propagation of cosmic rays in the solar wind. Rev. Geophys. Spa. Sci., 9, 27-87. 
Jørgensen, T. S., and A. W. Hansen 2000: Comment on "Variation of cosmic-ray flux and global cloud coverage - a missing link in solar-climate relationship" by Henrik Svensmark and Eigil Friis-Christensen [Journal of Atmospheric and Solar-Terrestrial Physics 59 (1997) 1225-1232]. J. Atmos. Terrest. Phys., 62, 73-77.

Kernthaler, S. C., R. Toumi, and J. D. Haigh 1999: Some doubts concerning a link between cosmic-ray fluxes and global cloudiness. Geophys. Res. Lett., 26, 863-865.

Kiehl, J. T., and B. P. Briegleb 1993: The relative roles of sulfate aerosols and greenhouse gases in climate forcing. Science, 260, 311-314.

Kirkby, J., and A. Laaksonen 2000: Solar variability and clouds. Space Sci. Rev., 94, 397-409.

Kuang, Z., Jiang, Y., and Y. K. Yung 1998: Cloud optical thickness variations during 1983-1991: Solar cycle or ENSO? Geophys. Res. Lett., 25, 1415-1417.

Labitzke, K. 1987: Sunspots, the QBO, and the stratospheric temperature in the north polar region. Geophys. Res. Lett., 14, 535-537.

Lean, J. 1997: The Sun's variable radiation and its relevance for Earth. Ann. Rev. Astron. Astrohys., 35, 33-67.

Lingenfelter, R. E. 1963: Production of carbon 14 by cosmic-ray neutrons, Rev. of Geophys., 1, 35-55.

Marsh, N. D., and H. Svensmark 2000: Low cloud properties influenced by cosmic rays. Phys. Rev. Lett., 85, 5004-5007.

McClatchey, R. A., R. W. Fenn, J. E. A. Selby, F. E. Volz, and J.S. Garing 1972: Optical properties of the atmosphere. Tech. Rep. AFCRL-72-0497, Air Force Cambridge Research Laboratories. 
Neher, H. V. 1961: Cosmic-ray knee in 1958. J. Geophys. Res., 66, 4007-4012.

1967: Cosmic-ray particles that changed from 1954 to 1958 to 1965. J. Geophys. Res., 72, 1527-1539.

Norris, J. R. 2000: What can cloud observations tell us about climate variability? Space Sci. Rev., 94, 375-380.

Pallé Bagó, E., and C. J. Butler 2000: The influence of cosmic rays on terrestrial clouds and global warming. Atstr. Geophys., 41, 4.18-4.22.

Pudovkin, M. I., and S. V. Veretenenko 1995: Cloudiness decreases associated with Forbush-decrease of Galactic cosmic rays. J. Atmos. Sol.-Terr. Phys., 57, 1349-1355.

Radke, L. F., Coakley, J. A. Jr., and M. D. King 1989: Direct and remote sensing observations of the effects of ships on clouds. Science, 246, 1146-1149.

Rasmusson, E. M., and T. M. Carpenter 1982: Variation in tropical sea surface temperature and surface wind fields associated with the Southern Oscillation/El Niño. Mon. Wea. Rev., 110, 354-384.

Reid, G. C. 1987: Influence of solar variability on global sea surface temperatures. Nature, 329, 142-143.

Reiter, R. 1992: Phenomena in Atmospheric and Environmental Electricity, Elsevier, 541 pp.

Ricchiazzi, P, S. Yang, and C. Gautier 1998: SBDART: a research and teaching software tool for plane-parallel radiative transfer in the Earth's atmosphere. Bull. Am. Met. Soc., 79, 2101-2114.

Rosenfeld, D. 2000: Suppression of rain and snow by urban and industrial air pollution. Science, 287, 1793-1796. 
Rossow, W. B., and R. A. Schiffer 1999: Advances in understanding clouds from ISCCP. Bull. Am. Met. Soc., 80, 2261-2287.

Stephens, G. L. 1978: Radiation profiles in extended water clouds II: parameterization schemes. J. Atmos. Sci., 35, 2123-2132.

1984: The parameterization of radiation for numerical weather prediction and climate models. Mon. Weath. Rev., 112, 826-867.

Svensmark, H., and E. Friis-Christensen 1997: Variation of cosmic ray flux and global cloud coverage - a missing link in solar-climate relationships. J. Atmos. Sol.-Terr. Phys., 59, 1225-1232.

Tinsley, B. A. 2000: Influence of solar wind on the global electric circuit, and inferred effects on cloud microphysics, temperature, and dynamics in the troposphere. Space Sci. Rev., 94, 231-258.

Tric, E. et al. 1992: Paleointensity of the geomagnetic field during the last 80,000 years. $J$. Geophys. Res., 97, 9337-9351.

Turco, R. P., Yu, F., and J.-X. Zhao 2000: Tropospheric sulfate aerosol formation via ion-ion recombination. J. Air $\&$ Waste Manage. Assoc., 50, 902-907.

Twomey, S. 1977: The influence of pollution on the shortwave albedo of clouds. J. Atmos. Sci., 34, 1149-1152.

van den Hulst, H. C. 1981: Light scattering by small particles. Dover Publications, 470 pp.

Wigley, T. M. L., and S. C. B. Raper 1992: Implications for climate and sea level of revised IPCC emission scenarios. Nature, 357, 293-300.

Wilson, C. T. R. 1901: On the ionization of atmospheric air. Proc.Roy. Soc. Lon., 68, 151-161. 
Yu, F. 2002: Altitude variations of cosmic-ray induced production of aerosols: Implications for global cloudiness and climate. J. Geophys. Res., in press.

Yu, F., and R. P. Turco 2000: Ultrafine aerosol formation via ion-mediated nucleation. Geophys. Res. Lett., 27, 883-886.

—, and — 2001: From molecular clusters to nanoparticles: role of ambient ionization in tropospheric aerosol formation. J. Geophys. Res., 106, 4797-4814.

Zhou, K., and C. J. Butler 1998: A statistical study of the relationship between the solar cycle length and tree-ring index values. J.Atmos. Sol.-Terr. Phys., 60, 1711-1718. 
Table 1. Temporal Fits to ISCCP Mean Cloud Opacity $(\bar{\tau})$ and Cloud Amount $(A)^{1}$

\begin{tabular}{ccccccc}
\hline \hline Par. & Lat. & Alt. & $\begin{array}{c}b_{0} \\
{[\text { Const. }}\end{array}$ & $\begin{array}{c}b_{1} \\
{[\mathrm{SOI}]}\end{array}$ & $\begin{array}{c}b_{2} \\
{[\text { Pinatubo] }}\end{array}$ & $\begin{array}{c}b_{3} \\
{[\mathrm{CR}]}\end{array}$ \\
\hline & & & & & & \\
$\bar{\tau}$ & $<40^{\circ}$ & High & $13.31 \pm 0.23$ & $7.6 \pm 1.7$ & $-32.2 \pm 1.6$ & $9.4 \pm 1.6$ \\
$\bar{\tau}$ & $<40^{\circ}$ & Mid-level & $10.45 \pm 0.32$ & $7.9 \pm 2.9$ & $-28.3 \pm 2.7$ & $5.8 \pm 2.9$ \\
$\bar{\tau}$ & $<40^{\circ}$ & Low & $3.78 \pm 0.14$ & $-1.9 \pm 3.1$ & $-4.2 \pm 3.6$ & $-2.5 \pm 3.4$ \\
$\bar{\tau}$ & $>40^{\circ}$ & High & $12.00 \pm 0.50$ & $16.6 \pm 4.0$ & $-6.9 \pm 3.6$ & $10.9 \pm 3.5$ \\
$\bar{\tau}$ & $>40^{\circ}$ & Mid-level & $6.81 \pm 0.23$ & $12.4 \pm 3.0$ & $-9.7 \pm 3.0$ & $1.8 \pm 3.2$ \\
$\bar{\tau}$ & $>40^{\circ}$ & Low & $5.01 \pm 0.14$ & $1.8 \pm 2.3$ & $-7.9 \pm 2.6$ & $-6.7 \pm 2.6$ \\
\hline$A$ & $<40^{\circ}$ & High & $13.84 \pm 0.52$ & $-4.8 \pm 0.32$ & $61.6 \pm 5.8$ & $-9.3 \pm 3.6$ \\
$A$ & $<40^{\circ}$ & Mid-level & $5.71 \pm 0.16$ & $2.0 \pm 2.5$ & $58.1 \pm 4.2$ & $-0.1 \pm 2.7$ \\
$A$ & $<40^{\circ}$ & Low & $45.5 \pm 1.6$ & $6.9 \pm 3.3$ & $13.6 \pm 4.0$ & $0.8 \pm 3.5$ \\
$A$ & $>40^{\circ}$ & High & $15.90 \pm 0.58$ & $-12.3 \pm 3.3$ & $7.9 \pm 4.0$ & $-9.9 \pm 3.3$ \\
$A$ & $>40^{\circ}$ & Mid-level & $23.61 \pm 0.84$ & $-4.6 \pm 3.0$ & $8.3 \pm 3.8$ & $-3.1 \pm 3.4$ \\
$A$ & $>40^{\circ}$ & Low & $38.7 \pm 1.0$ & $7.1 \pm 2.4$ & $10.1 \pm 2.9$ & $6.5 \pm 2.6$
\end{tabular}

${ }^{1}$ Except for the amplitude of the constant term $b_{0}$, the model amplitudes for the SOI, Mt. Pinatubo, and cosmic-ray terms $\left(b_{1}-b_{3}\right.$, respectively) are normalized to $b_{0}$ and expressed as a percentage. The mean cloud opacity $\bar{\tau}$ and total mean amount fraction $A$ are from the ISCCP visible band data. 
Fig. 1. - Cartoon illustrating two limiting scenarios for the effect of the Galactic cosmic rays (GCRs) on cloud optical properties, assuming that the varying ionizing cosmic-ray flux causes changes in the number of cloud condensation nuclei $(\mathrm{CCN})$ through ion-mediated nucleation. In the first case we assume that the nucleation of cloud droplets is limited by the available amount of water in the supersaturated air. Therefore, as illustrated in the top panel, if an increase in the GCR ionization flux resulted in more cloud condensation nuclei (CCN) but no additional water condensation, the amount of water per droplet will be less and the effective radius $R_{\text {eff }}$ of the droplet distribution will be smaller. Alternately, as illustrated in the bottom panel, if the formation of cloud droplets is limited by the local availability of CCN and not condensible water, $R_{\text {eff }}$ can remained unchanged and additional CCN resulting from changes in cosmic-ray ionization would cause an increase in the amount of water extracted from the supersaturated air, so the cloud liquid water content would increase. The opposite trends hold for cases where the number of CCN is decreased by variations in the cosmic-ray flux.

Fig. 2.- The fractional change in the albedo of a $1 \mathrm{~km}$ thick cloud expected from a $10 \%$ increase in the number of cloud droplets due to changes in the cosmic-ray flux, shown for the case of variable cloud water content LWC (top) and for variable droplet radius $R_{\text {eff }}$ (bottom). The solid contours denote the change in albedo, and the dotted contours are for the optical thickness.

Fig. 3. - The fractional change in the albedo, expected from a $10 \%$ increase in the number of cloud droplets from variations in the cosmic-ray rate, plotted as a function of cloud optical thickness for three different cloud geometrical thicknesses. The open symbols denote changes in cloud LWC and the filled symbols changes in $R_{e f f}$. 
Fig. 4.- The mean cloud $0.6 \mu \mathrm{m}$ optical thickness from the ISCCP database for all clouds in the low latitude band $|\phi|<40^{\circ}$, with the cosmic-ray rate from the Climax neutron monitor. The high, mid-level, and low clouds refer to cloud top pressures of $P<440 \mathrm{mb}$, $440<P<680 \mathrm{mb}$, and $P>680 \mathrm{mb}$, respectively, and the shaded interval refers to cloud data affected significantly by the eruption of Mt. Pinatubo in June 1991. The $1 \sigma$ error bars on the ISCCP data were calculated from the sample variance of the data.

Fig. 5. - Same as Figure 4, but for all high latitude clouds with $|\phi|>40^{\circ}$.

Fig. 6.- The mean cloud $0.6 \mu \mathrm{m}$ amount fractions from the the ISCCP database for all clouds in the low latitude band $|\phi|<40^{\circ}$, with the cosmic-ray rate from the Climax neutron monitor. The $1 \sigma$ error bars on the ISCCP data were calculated from the sample variance of the data.

Fig. 7.- Same as Figure 6, but for all high latitude clouds with $|\phi|>40^{\circ}$.

Fig. 8.- Basis vectors used in the temporal model of ISCCP visible opacity and amount fraction variations. The vectors $X_{0}, X_{1}, X_{2}$, and $X_{3}$ represent the constant level term and variations due to ENSO, the eruption of Mt. Pinatubo, and cosmic rays, respectively.

Fig. 9.- Fractional change in visible cloud amount versus fractional change in visible opacity, from the fit of the temporal model to the ISCCP data. The points have been normalized by their respective constant term values in the temporal model, and the error bars have been omitted. In all cases there is an inverse correlation between the two parameters. 


\section{Solar Max. \\ (low GCR flux)}

\section{Solar Min. \\ (high GCR flux)}

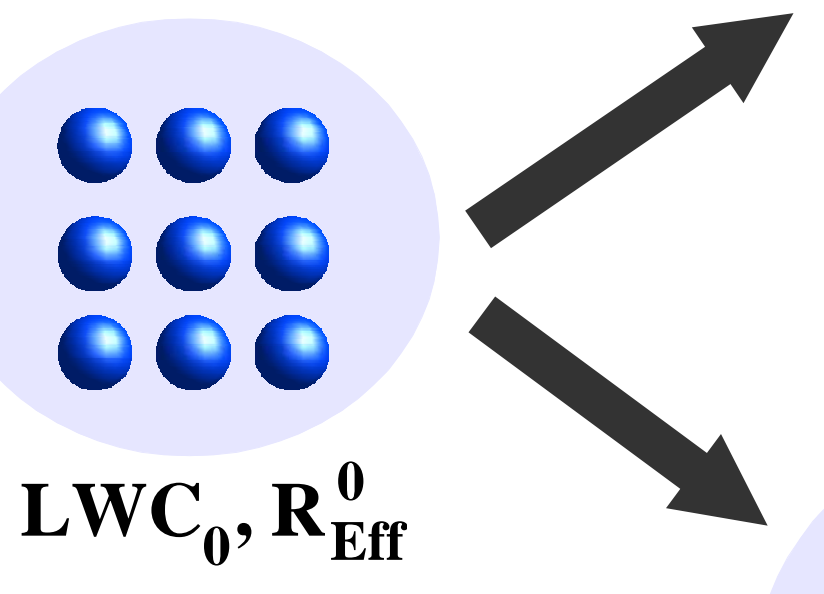

Droplet in Cloud

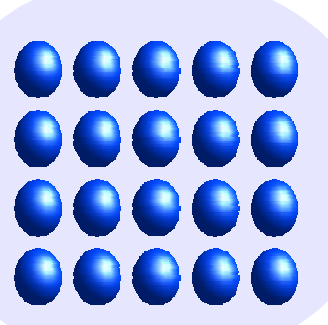

Case 1:

More CCN

$\mathrm{LWC}=\mathrm{LWC}_{0}$

$\mathbf{R}_{\text {Eff }}<\mathbf{R}_{\text {Eff }}^{\mathbf{0}}$
Case 2:

More Water

$\mathbf{R}_{\text {Eff }}=\mathbf{R}_{\text {Eff }}^{\mathbf{0}}$

$\mathbf{L W C}>\mathbf{L W C}_{\mathbf{0}}$ 


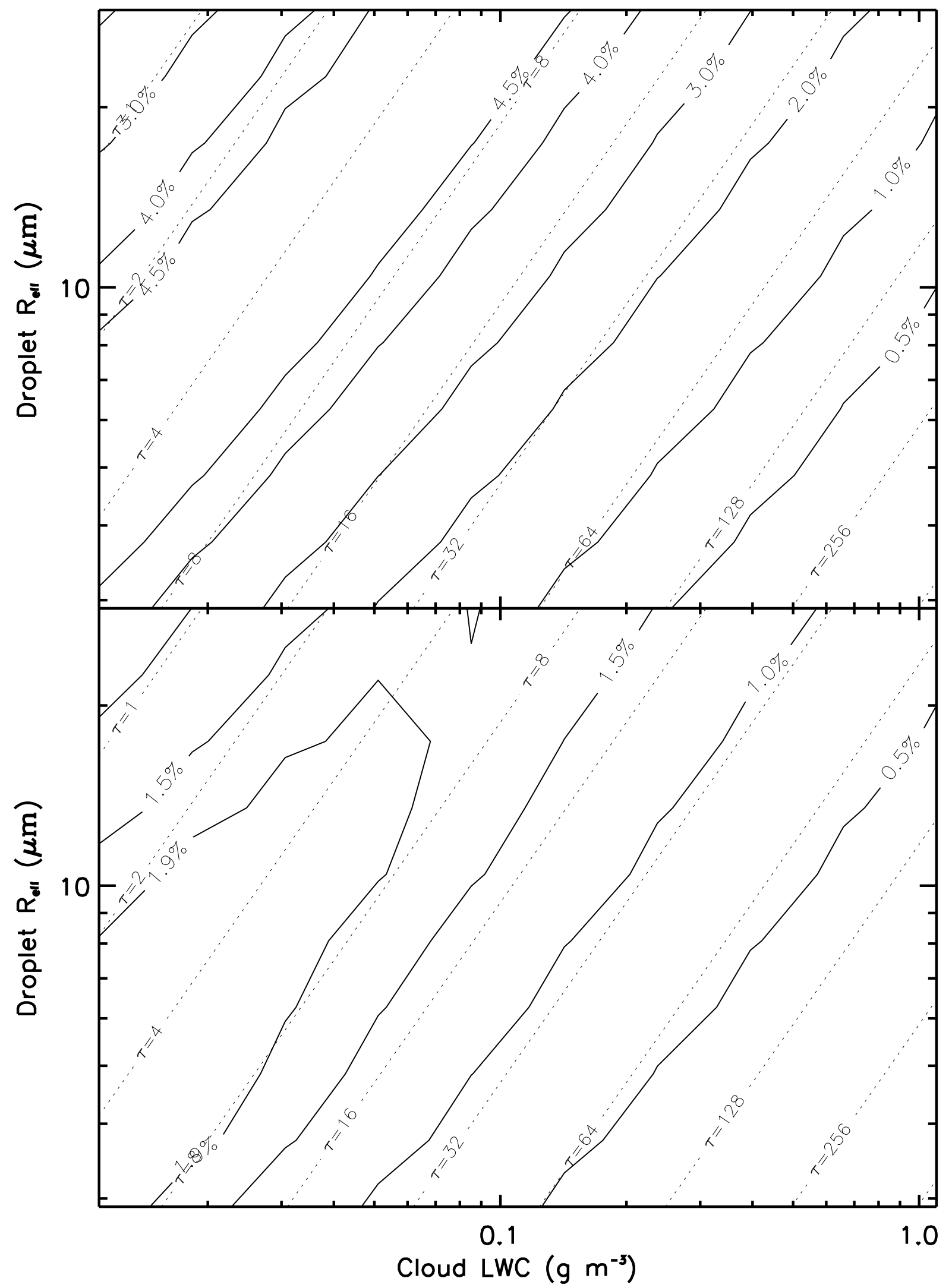




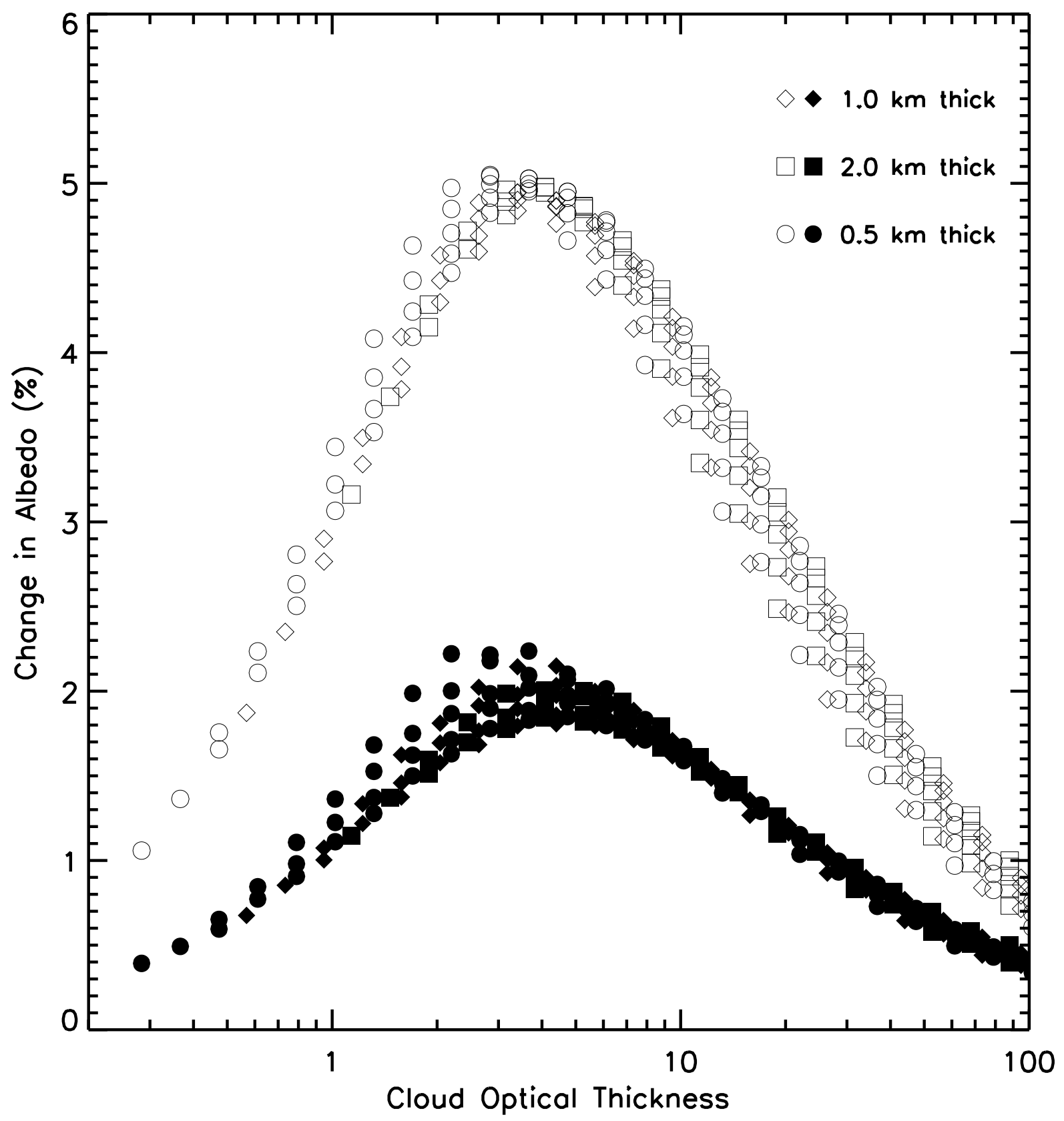




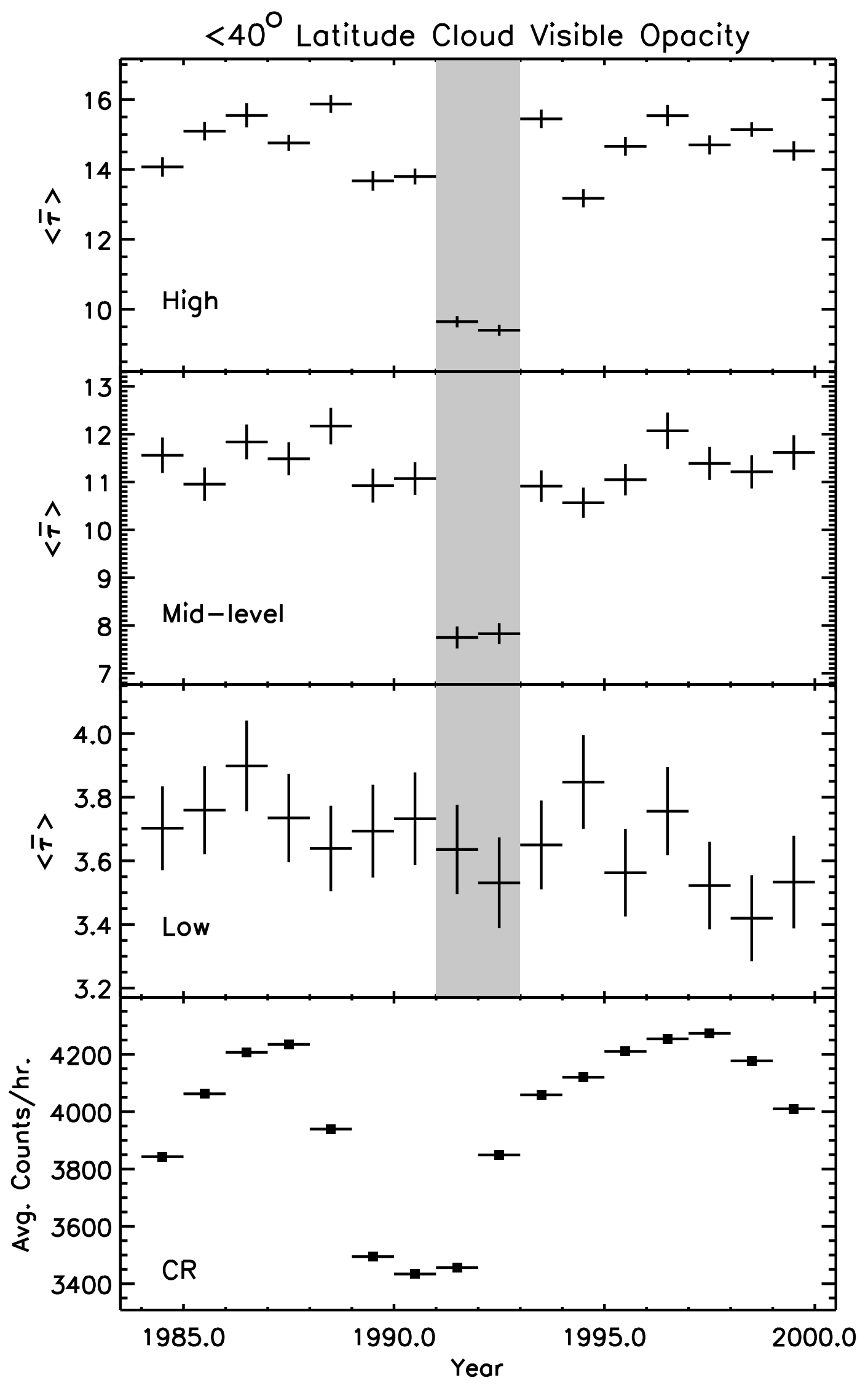




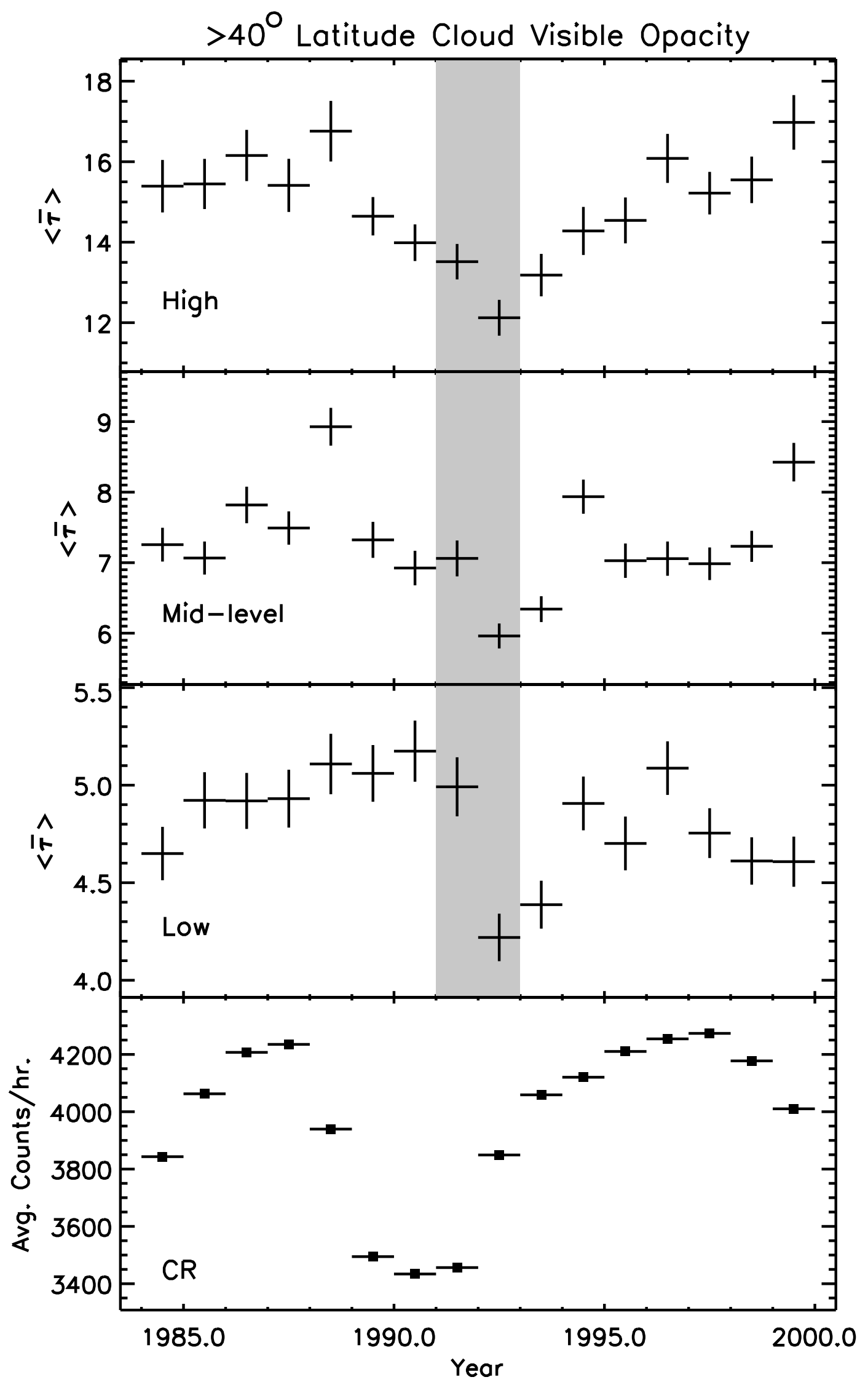




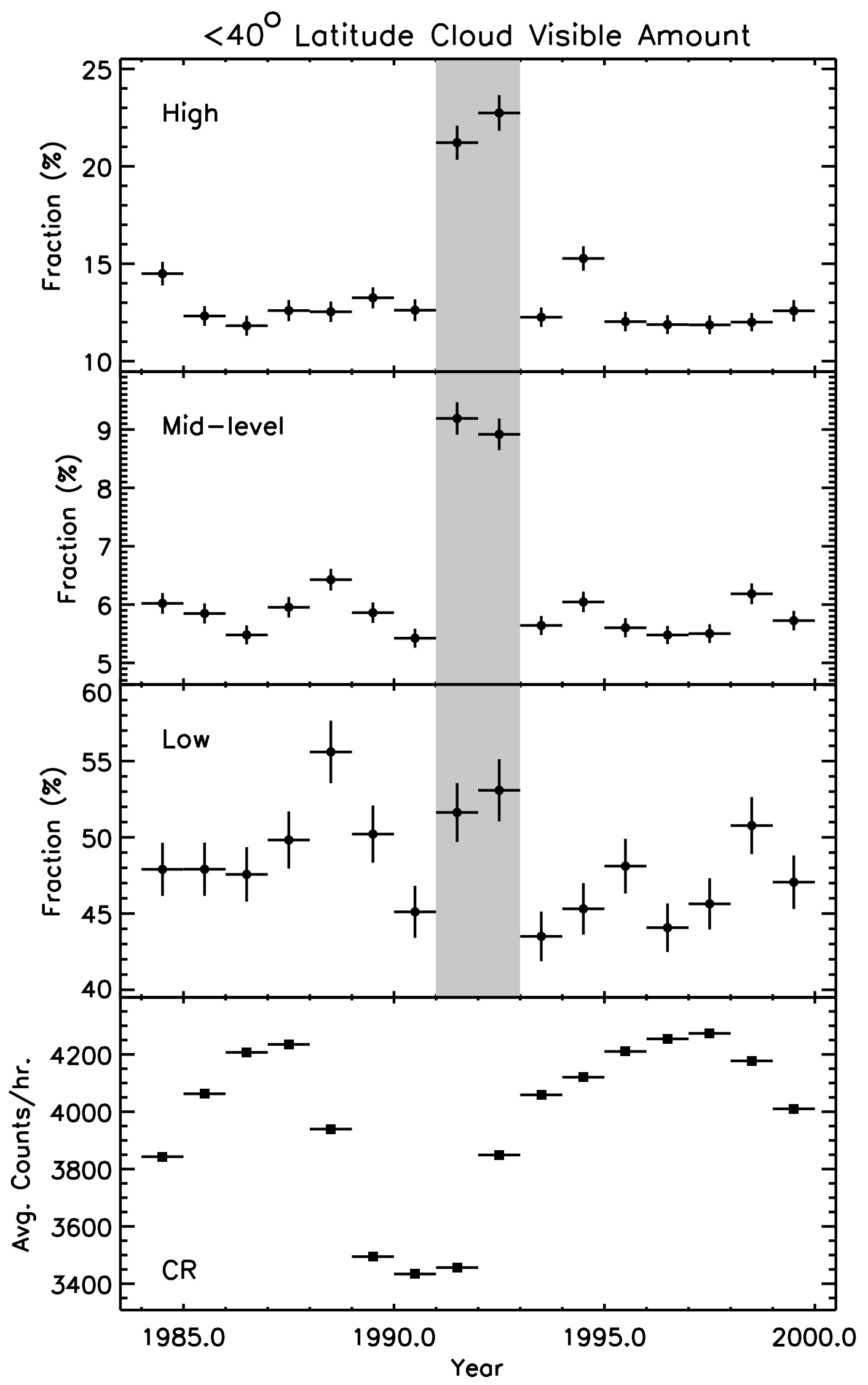




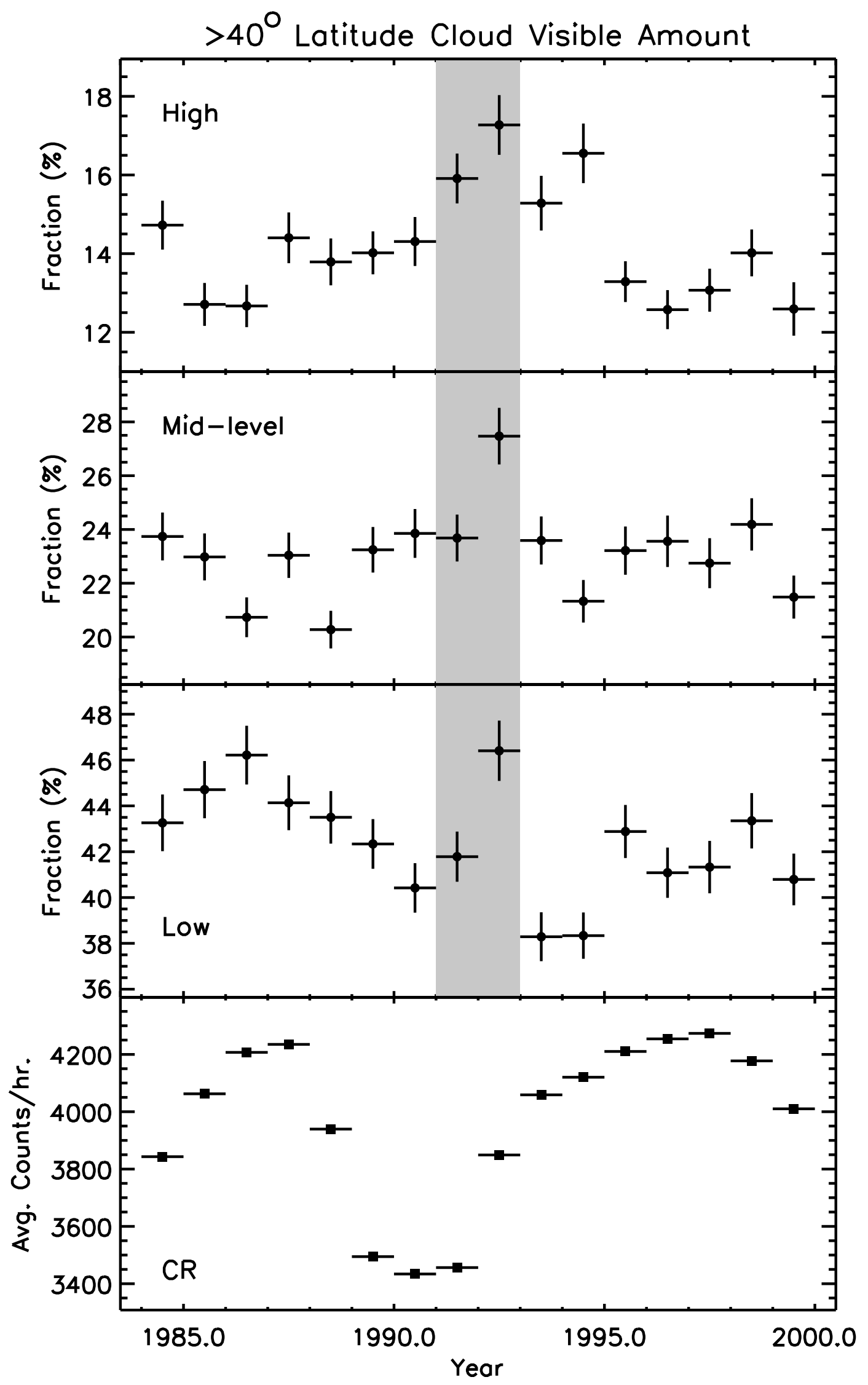




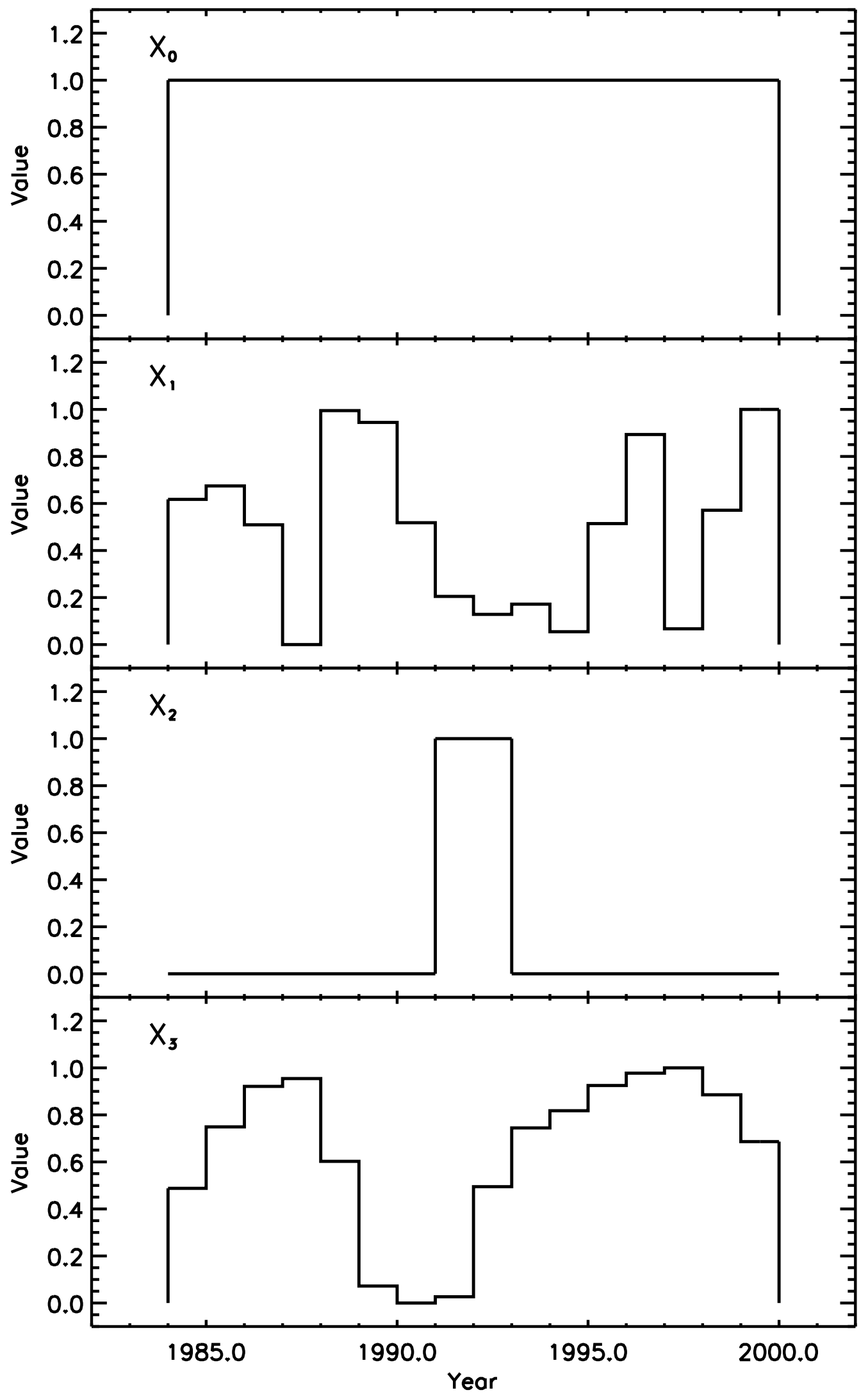



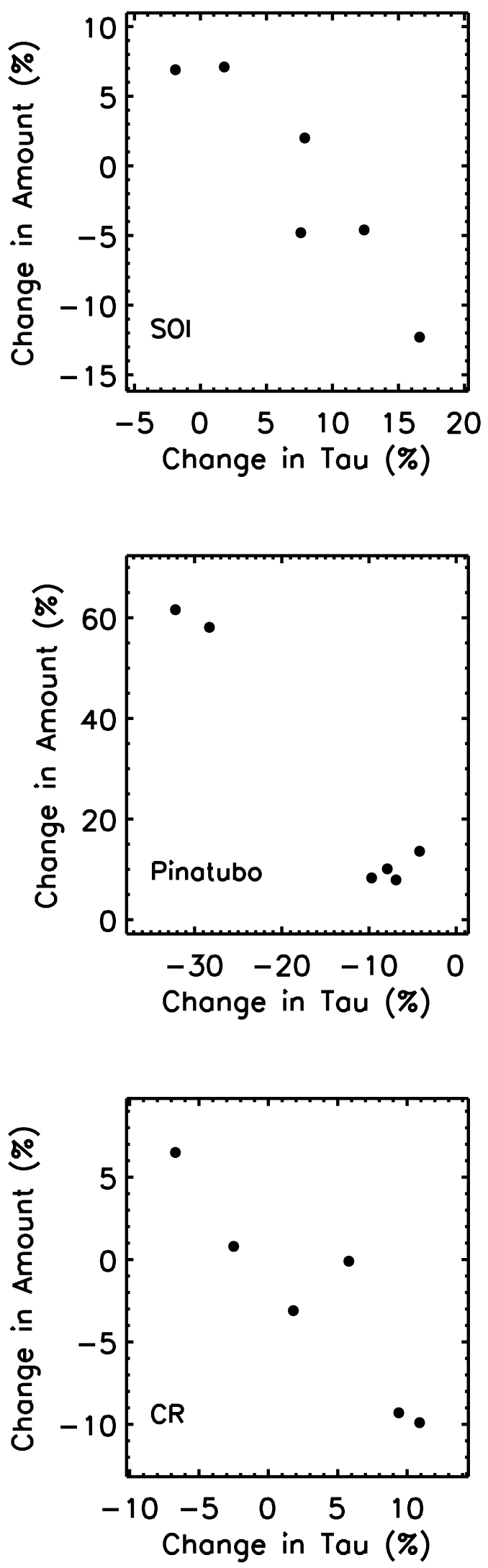